\title{
Cost-Effectiveness of Hepatitis B Mass Screening and Management in High-Prevalent Rural China: A Model Study From 2020 to 2049
}

\author{
Xiaolan $\mathrm{Xu}^{1{ }^{1 \oplus}}{ }^{\oplus}$, Chensi Wu ${ }^{1 \uparrow}{ }^{\circledR}$, Lushun Jiang $^{1}$, Chunting Peng ${ }^{1}$, Liya Pan ${ }^{1}$, Xue Zhang ${ }^{1}$, Wei Shen ${ }^{1}$, Lin Chen ${ }^{1}$, \\ Zhuoqi Lou ${ }^{1}$, Kaijin $\mathrm{Xu}^{1}$, Lanjuan $\mathrm{Li}^{1}$, Yin Dong ${ }^{2 *}$, Bing Ruan ${ }^{{ }^{*} \mathbb{D}}$
}

\begin{abstract}
Background: Chronic hepatitis $\mathrm{B}(\mathrm{CHB})$ is highly prevalent among adults in rural China and better management of those populations is of vital importance for viral hepatitis elimination. Adult immunization has been the subject of much controversy in previous studies. This study estimates the cost-effectiveness of population-based hepatitis B screening, treatment, and immunization strategy (comprehensive strategy) in rural areas with high prevalence under the national policy of sharp-drop drug prices.

Methods: We constructed a Markov model comparing 4 strategies in a 30-year horizon from the healthcare payer perspective: (1) the conventional pattern; (2) screening and treating infected (treatment); (3) screening and immunizing susceptible individuals (immunization); and (4) the comprehensive strategy. Screening intensity ranged from $50 \%$ to $100 \%$. Outcomes were measured by costs, quality-adjusted life-years (QALYs), incremental cost-effectiveness ratios (ICERs), and clinical outcomes.

Results: The costs for the conventional pattern, treatment strategy, immunization strategy, and comprehensive strategy were US\$341, 351, 318, and 323, respectively. In addition, effects were 17.45, 17.57, 17.46, and 17.58 QALYs, respectively. The ICER of the comprehensive strategy was US\$35/QALY gained at 50\% screening intensity and 420 US\$/QALY gained at $100 \%$. The net monetary benefit increased with increasing screening intensity and declined after $90 \%$, with the highest value of US $\$ 40693$. All new infections and 52.5\% mortality could be avoided from 2020 to 2049 if all patients were properly treated and all susceptible individuals were immunized. The results were stable within a wide range of parameters.

Conclusion: It was cost-effective to implement the mass hepatitis B screening, treatment, and immunization strategy in areas of rural China with high prevalence, and the strategy gained the most net monetary benefit at a screening intensity of $90 \%$. Although it was impractical to fulfill $100 \%$ coverage, efforts should be made to obtain more people screened.

Keywords: Economic Evaluation, Viral Hepatitis, Antiviral Treatment, Adult Vaccination

Copyright: @ 2022 The Author(s); Published by Kerman University of Medical Sciences. This is an open-access article distributed under the terms of the Creative Commons Attribution License (https://creativecommons.org/licenses/ by/4.0), which permits unrestricted use, distribution, and reproduction in any medium, provided the original work is properly cited.

Citation: $\mathrm{Xu} \mathrm{X}, \mathrm{Wu} \mathrm{C}$, Jiang $\mathrm{L}$, et al. Cost-effectiveness of hepatitis B mass screening and management in high-prevalent rural china: a model study from 2020 to 2049. Int J Health Policy Manag. 2022;11(10):21152123. doi:10.34172/ijhpm.2021.126
\end{abstract}

\section{Article History:}

Received: 29 March 2021

Accepted: 4 September 2021

ePublished: 7 September 2021

IBoth authors contributed equally to this paper.

\section{*Correspondence to: \\ Yin Dong}

Email: 9597082@qq.com

Bing Ruan

Email: ruanbing@zju.edu.cn

\section{Key Messages}

Implications for policy makers

- Population-based mass hepatitis B virus (HBV) screening, treatment, and immunization strategy for rural residents in high prevalence areas is cost-effective.

- The adult HBV immunization strategy is cost-saving if fewer than $90 \%$ of susceptible individuals are vaccinated, and new infection could be reduced by nearly $100 \%$ by universal immunization. Free vaccines for adults could be considered.

- Early detection and treatment of HBV infection, and vaccination for susceptible persons is the route we must take to realize HBV elimination by 2030 .

Implications for the public

This study proves that population-based hepatitis B virus (HBV) screening, treatment, and immunization strategy in rural areas with high prevalence will prevent most new hepatitis B infections if all susceptible individuals are vaccinated. Although it is impractical to make every person get the vaccination, we should endeavor to reach $90 \%$ participation in the population. However, there are many HBV patients currently in China. The prevalence is high, especially in the middle-aged and elderly population, because they were not vaccinated. Complications such as liver cirrhosis and hepatocellular carcinoma (HCC) are prone to occur in this population, so mortality decreases slightly (52.5\% in this study). Therefore, it is necessary to increase public willingness to participate in HBV screening to increase the detection rate and offer early management and treatment to reduce incidence and mortality. Therapeutic efficiency should be enhanced for patients in progressive stages. 


\section{Background}

A total of 257 million people were infected with hepatitis B virus (HBV) by 2015 globally, leading to nearly $900000 \mathrm{HBV}-$ related deaths. ${ }^{1}$ Although the HBV prevalence in children under 5 years of age has dropped to $0.3 \%$ in China, there were nearly 100 million HBV infections in $2015 .{ }^{2,3}$ Only $19 \%$ of those have been diagnosed, and $10 \%-11 \%$ of those with treatment indications have initiated antiviral treatment, which was far from the World Health Organization (WHO) targets of $90 \%$ of $\mathrm{HBV}$-infected diagnosed and $80 \%$ on treatment. ${ }^{3}$ A model study predicted that 60 million HBV infections and 680000 HBV-related deaths would occur in China by 2030 with current practice. ${ }^{4}$

Long-term treatment could postpone the occurrence of liver cirrhosis or HBV-related hepatocellular carcinoma (HCC) to improve quality of life. ${ }^{5} \mathrm{HBV}$ screening and treatment were cost-effective in community-based study in West Africa or with immigrants in North America. ${ }^{6,7}$ Immunization was the most effective way to prevent new infections. The immunization rate in 2006 was less than $10 \%$ in susceptible individuals aged 15 59 in China. ${ }^{8}$ Moreover, approximately $40 \%$ of those who received immunization at birth had an $\mathrm{HBV}$ surface antibody (HBsAb) level below $10 \mathrm{mIU} / \mathrm{mL}$ in adolescence, and it was economically beneficial to vaccinate close contacts, individuals with diabetes, immigrants, men who have sex with men, sex workers, incarcerated people, and drug abusers. ${ }^{9-13}$

HBV prevalence in 2006 in the rural population and unvaccinated individuals aged $1 \sim 59$ years was $7.3 \%$ and $9.4 \%$, respectively. ${ }^{14}$ Since China issued the "National Organization of Drug Centralized Procurement and Use Pilot Program" in 2019 , the price of antiviral drugs has decreased sharply, which may greatly mitigate the economic burden of treatment to rural patients. Thus, we carried out an economic evaluation of whether mass screening, treatment, and immunization could be cost-effective in this population with various intervention intensities in the next 30 years.

\section{Methods}

\section{Model Structure}

We used TreeAge Pro Healthcare (TreeAge Software, Inc., Williamstown, Massachusetts) to build a Markov decision tree model. The progression after infection was divided into 10 states: immune acquired, immune tolerant, acute hepatitis $\mathrm{B}$, chronic hepatitis $\mathrm{B}(\mathrm{CHB})$, inactive $\mathrm{HBV}$ surface antigen (HBsAg) carrier, compensated liver cirrhosis, decompensated liver cirrhosis, HBV-related HCC, liver transplantation, and death. ${ }^{15}$ As the disease progressed, transitions occurred between the above disease states. The transition probability was obtained from the published literature (Table 1), and the range was represented by the $95 \%$ confidence interval (CI). The maximum and minimum determined in the literature were replaced as the variation range if the $95 \%$ CI was absent. If there was only one value available, the variation range was set to $\pm 25 \%$ and constrained between 0 and 1 . With 2020 as the entry year, the Markov model ran 30 cycles with a 1-year interval, simulating HBV epidemiology in a fixed cohort. The model was constructed based on the following assumptions and detailed in Supplementary file 1:

1. Except for the mutual transition between $\mathrm{CHB}$ and inactive HBsAg carriers, the transition of other disease states was irreversible (Figure S1, Supplementary file 1), ${ }^{16}$ and the transition probability between each state was fixed each year.

2. Individuals with $\mathrm{HBs} A b$ titers above $10 \mathrm{mIU} / \mathrm{mL}$ would not be infected with HBV.

3. In the conventional pattern, rural patients did not go to a hospital for health examinations on their initiative until exacerbation. While symptoms were obscure in carriers and $\mathrm{CHB}$ patients who were discovered in the later stage in most cases, it was assumed that there was no treatment cost in these groups in the conventional pattern. In addition, we hypothesized that comprehensive management of HBV carriers with regular follow-up could reduce their incidence of $\mathrm{CHB}$, liver cirrhosis, and HCC by $50 \%$.

4. The simplified model simulating the natural history of $\mathrm{HBV}$ infection and the altered progress after antiviral treatment or immunization was depicted in Figure S2 (Supplementary file 1).

\section{Cohort Characteristics}

HBV prevalence in rural coastal areas in Zhejiang province was reported to be high in a community-based survey, ranging from $7.5 \% \sim 17.0 \%$ in different age groups, especially in those aged 20 60, and decreased among people over 60 years old. ${ }^{33,34}$ We selected rural residents aged 28 60 years for modeling. We assumed that this population had never been vaccinated because a free HBV neonatal immunization program was launched in 1992. HBsAg prevalence in this age group was analyzed by serum samples collected during HBV screening in 2019 (Table S1, Supplementary file 2). The costs, probabilities, and outcomes involved in this model were set to be independent of age and gender.

\section{Comparators}

Based on current HBV screening, we compared the treatment and immunization strategy (comprehensive) with: (1) treatment and management for HBsAg positive individuals (treatment); (2) immunization for susceptible individuals (HBsAg and HBsAb negative) (immunization); and (3) the conventional pattern. No intervention was performed in the conventional pattern, and the population was not screened for $\mathrm{HBV}$ infection, so individuals living with $\mathrm{HBV}$ may not be found in a timely manner. They do not usually go to the hospital until severe symptoms develop, often caused by liver cirrhosis or HCC. This may cause a delay in the timing of treatment; additionally, hidden patients are not conducive to the control of new infections. We screened HBV serological markers in the immunization strategy and HBV DNA quantification, liver function, and liver ultrasonography additionally in the other two strategies. Immunization was carried out in the first year of the simulation cohort.

We have carried out HBV screening in our demonstration areas since 2009 , and more than $50 \%$ of rural residents participated in the screening (more details are provided in 


\begin{tabular}{|c|c|c|c|c|}
\hline & Base Value & Deterministic Range & PSA Distribution & Reference \\
\hline \multicolumn{5}{|l|}{ General inputs } \\
\hline Start age of cohort, y & 28 & $28-60$ & - & - \\
\hline Discount rate, \% & 3.00 & $0.00-6.00$ & Uniform & 6 \\
\hline HBsAg positive rate, $\%$ & 11.10 & $10.60-11.50$ & Beta & Supplementary file 2 \\
\hline HBsAb positive rate, $\%$ & 60.50 & $59.80-61.20$ & Beta & Supplementary file 2 \\
\hline Screening intensity, \% & 50.00 & $50.00-100.00$ & - & Assumption \\
\hline Seroconversion rate after immunization, \% & 91.50 & $88.00-95.00$ & Beta & 17 \\
\hline \multicolumn{5}{|l|}{ Cost inputs } \\
\hline Annual cost for acute infection, US\$ & 1531.80 & - & Gamma & Supplementary file 2 \\
\hline Annual cost for compensated cirrhosis, US\$ & 1252.30 & - & Gamma & Supplementary file 2 \\
\hline Annual cost for decompensated cirrhosis, US\$ & 3441.30 & - & Gamma & Supplementary file 2 \\
\hline Annual cost for HCC, US\$ & 2267.20 & - & Gamma & Supplementary file 2 \\
\hline Annual cost for transplantation (first year, US\$) & $45748.00^{\mathrm{a}}$ & - & Gamma & 18 \\
\hline Annual cost for transplantation (subsequent year, US\$) & $7926.00^{\mathrm{a}}$ & - & Gamma & 18 \\
\hline Annual cost for intervening carriers, US\$ & 75.70 & - & Gamma & Supplementary file 2 \\
\hline Annual cost for intervening $\mathrm{CHB}$, US\$ & 205.30 & - & Gamma & Supplementary file 2 \\
\hline Immunization cost, US\$ & 50.40 & - & Gamma & Supplementary file 2 \\
\hline Screening cost for immunization, US\$ & 10.90 & - & - & Supplementary file 2 \\
\hline Screening cost for treatment, US\$ & 29.10 & - & Gamma & Supplementary file 2 \\
\hline \multicolumn{5}{|l|}{ Annual disease transition rates } \\
\hline From immunotolerant to immune active, $\%$ & 3.40 & $2.55-4.25$ & Beta & 19 \\
\hline From CHB to inactive carrier, $\%$ & 5.70 & $4.58-6.88$ & Beta & 6 \\
\hline Treated CHB to inactive carrier, \% & 12.60 & $11.40-13.80$ & Beta & 12 \\
\hline From CHB to compensated cirrhosis, $\%$ & 3.20 & $2.40-4.00$ & Beta & 20 \\
\hline Treated CHB to compensated cirrhosis, $\%$ & 1.76 & $1.32-2.20$ & Beta & 20,21 \\
\hline From $\mathrm{CHB}$ to $\mathrm{HCC}, \%$ & 0.60 & $0.53-0.72$ & Beta & 16 \\
\hline Treated CHB to HCC, \% & 0.30 & $0.25-0.35$ & Beta & 16,21 \\
\hline From $\mathrm{CHB}$ to death, $\%$ & 0.75 & $0.00-1.50$ & Beta & 20 \\
\hline Treated CHB to death, $\%$ & 0.45 & $0.00-0.90$ & Beta & 20,21 \\
\hline From inactive carrier to $\mathrm{HBsAg}$ clearance, $\%$ & 1.00 & $0.00-2.00$ & Beta & 16 \\
\hline Treated inactive carrier to HBsAg clearance, \% & 4.50 & $2.00-7.00$ & Beta & 22 \\
\hline From inactive carrier to $\mathrm{CHB}, \%$ & 4.40 & $1.60-4.70$ & Beta & 6,23 \\
\hline From inactive & 0.10 & $0.08-0.12$ & Beta & 12 \\
\hline From inactive carrier to compensated cirrhosis, $\%$ & 0.10 & $0.00-0.10$ & Beta & 16 \\
\hline From compensated cirrhosis to decompensated cirrhosis, $\%$ & 4.00 & $3.00-4.00$ & Beta & 16 \\
\hline Treated compensated cirrhosis to decompensated cirrhosis, \% & 1.80 & $1.35-1.80$ & Beta & 16,21 \\
\hline From cirrhosis to $\mathrm{HCC}, \%$ & 3.70 & $3.10-4.14$ & Beta & 16 \\
\hline Treated cirrhosis to HCC, \% & 2.00 & $1.40-4.10$ & Beta & 24 \\
\hline From compensated cirrhosis to death, $\%$ & 3.40 & $2.90-3.90$ & Beta & 6,16 \\
\hline Treated compensated cirrhosis to death, $\%$ & 1.10 & $0.70-1.60$ & Beta & 25 \\
\hline From decompensated cirrhosis to death, $\%$ & 26.50 & $21.40-31.60$ & Beta & 16 \\
\hline Treated decompensated cirrhosis to death, \% & 8.60 & $7.50-9.70$ & Beta & 26 \\
\hline From decompensated cirrhosis to liver transplantation, \% & 0.65 & $0.49-0.81$ & Beta & 27 \\
\hline From HCC to death, $\%$ & 14.40 & $11.60-17.20$ & Beta & 20 \\
\hline From HCC to liver transplantation, $\%$ & 0.65 & $0.49-0.81$ & Beta & 27 \\
\hline Annual mortality rate after liver transplantation, $\%$ & 7.80 & $4.90-10.70$ & Beta & 28 \\
\hline Annual natural mortality rate, $\%$ & $0.71^{\mathrm{b}}$ & $0.53-0.89$ & Beta & - \\
\hline From susceptible to infected, $\%$ & 0.95 & $0.71-1.19$ & Beta & 29 \\
\hline From acute infected to $\mathrm{CHB}, \%$ & 5.00 & $1.00-10.00$ & Beta & 30,31 \\
\hline From acute infected to death, \% & 0.80 & $0.30-1.70$ & Beta & 12 \\
\hline
\end{tabular}


Table 1. Continued

\begin{tabular}{|c|c|c|c|c|}
\hline & Base Value & Deterministic Range & PSA Distribution & Reference \\
\hline \multicolumn{5}{|l|}{ Utility parameters } \\
\hline Immune acquired & 0.99 & $0.98-1.00$ & Beta & 12 \\
\hline Susceptible & 0.99 & $0.98-1.00$ & Beta & 12 \\
\hline Immune tolerant & 0.99 & $0.98-1.00$ & Beta & 12 \\
\hline Acute hepatitis B & 0.70 & $0.63-0.77$ & Beta & 12 \\
\hline $\mathrm{CHB}$ & 0.52 & $0.47-0.57$ & Beta & 32 \\
\hline Inactive HBsAg carrier & 0.85 & $0.77-0.93$ & Beta & 12 \\
\hline Compensated cirrhosis & 0.57 & $0.52-0.62$ & Beta & 32 \\
\hline Decompensated cirrhosis & 0.26 & $0.21-0.31$ & Beta & 32 \\
\hline $\mathrm{HCC}$ & 0.31 & $0.26-0.36$ & Beta & 32 \\
\hline Liver transplantation (first year) & 0.41 & $0.36-0.46$ & Beta & 32 \\
\hline
\end{tabular}

Abbreviations: PSA, probabilistic sensitivity analysis; HBsAb, HBV surface antibody; HCC, hepatocellular carcinoma; CHB, chronic hepatitis B.

a The method used to obtain the data are explained in "Cost estimate" in Supplementary file 2.

${ }^{\mathrm{b}}$ Referred from China Population \& Employment Statistics Yearbook 2019.

part 3 of Supplementary file 2). Therefore, we hypothesized that the basic screening intensity was 50\%. Given the actual situation, the higher the screening intensity, the greater the benefits gained, but the intervention cost might increase nonlinearly. Cost increased by $50 \%$ with each additional $10 \%$ screened. Thus, different screening intensities were simulated and the cost-effectiveness could be calculated to explore the best screening strategy suitable for local conditions.

Referring to the Guideline of Prevention and Treatment for Chronic Hepatitis B in China, we formulated a treatment and follow-up scheme for each disease state: (1) all HBV infected states except for immune tolerant, acute hepatitis B, and inactive HBsAg carriers should take antiviral drugs; (2) immune tolerant and inactive HBsAg carriers were followed up every six months for routine blood tests, biochemical tests, HBV serological markers, alpha fetoprotein, liver ultrasonography and elastography; (3) patients treated with nucleos(t)ide analogs were followed up every three months for routine blood tests, biochemical tests, HBV DNA quantification, and HBV serological markers; and (4) patients with cirrhosis should be inspected for alpha fetoprotein, liver ultrasonography and elastography every three months, and patients without cirrhosis were inspected for the above items every six months.

\section{Estimating Costs}

The costs were divided into four components: screening costs, immunization costs, costs related to health states, and treatment costs. The cost of the screening items referred to the pricing of local secondary hospitals (Table S2, Supplementary file 2). The immunization costs included vaccine fees and inoculation service fees. For costs related to health states, we only calculated direct medical expenses (including registration fees, drug fees, diagnosis and treatment fees, hospitalization fees, examination fees, etc) in the model (more details for costs are provided in Supplementary file 2). We calculated the outpatient expenses under each disease state separately according to the Guideline of Prevention and Treatment for Chronic Hepatitis B in China, and the hospitalization expenses were obtained from previous literature and the electronic medical record system of the People's Hospital of Yuhuan county (Table S3, Supplementary file 2). For treatment costs, the cost of standard follow-up and treatment for the screened inactive carriers and $\mathrm{CHB}$ patients was considered. We calculated the total cost from the healthcare payer perspective. Costs and health benefits were discounted at a discount rate of $3 \%$ per year. ${ }^{6}$ All costs are presented in US\$, with an exchange rate of US\$1 $=6.8985$ China Yuan in 2019.

\section{Outcome Measurement and Cost-Effectiveness}

Outcomes were presented by (1) average costs; (2) average life-years; (3) average quality-adjusted life-years (QALYs); and (4) clinical outcomes including new HBV infections, compensated or decompensated cirrhosis, HBV-related HCC, liver transplantation and mortality. Utilities for hepatitisrelated health states elicited from mainland China patients in the study of Levy et al were adopted. ${ }^{32}$ The range of health utilities varied by 0.05 if a $95 \%$ CI was lacking. The incremental cost-effectiveness ratio (ICER) was used to compare the economic feasibility of each intervention, which meant the cost needed to gain an additional QALY. An intervention was supposed to be cost-effective with an ICER lower than the willingness-to-pay (WTP) threshold. The WHO recommends that an ICER less than three times the annual gross domestic product (GDP) per capita is cost-effective, while less than one times the GDP per capita is extremely cost-effective. ${ }^{35}$ The GDP per capita in 2019 was US\$10 276 in China. ${ }^{36}$ Given the rural population selected in our study, we adopted a more rigorous criterion, the disposable income of rural residents per capita in 2019 (US\$2322), as the appointed WTP.

Sensitivity Analysis

The parameters involved in the model varied within wide 
ranges. We performed a one-way sensitivity analysis of the screening intensity to determine the most appropriate intensity. Then, we performed a one-way sensitivity analysis for all parameters at the preferred intensity and presented the results through a tornado diagram. The variation range of the costs was set to $\pm 50 \%$. A two-way sensitivity analysis was conducted to determine the preferred strategy when the screening costs in the comprehensive strategy and screening intensity were altered synchronously. Multivariate probabilistic sensitivity analysis (PSA) reflected the probability that interventions were cost-effective within the WTP threshold when all parameters changed simultaneously. Distributions for parameters were specified by gamma distributions for costs (range of $\pm 50 \%$ ), beta distributions for probabilities, epidemiological parameters, and utilities, and uniform distributions for discount rate and screening intensity. The beta distributions were derived by the calculation method with the mean and standard deviation. The mean was the base value in Table 1, and the standard deviation was half of the difference of the upper and lower limits of the deterministic range. The PSA results were presented in the form of a costeffectiveness acceptability curve.

\section{Results}

Costs, QALYs, and Cost-Effectiveness at Baseline Intensity The model has been validated to be consistent with realworld epidemic data (Table S4, Supplementary file 1). In the conventional pattern, the average cost per person was US $\$ 341$ and obtained 18.08 life-years and 17.45 QALYs, respectively. In the treatment strategy, the average cost was US $\$ 351$ and obtained 18.12 life-years and 17.57 QALYs, respectively. In the immunization strategy, the average cost was US $\$ 318$ and obtained 18.08 life-years and 17.46 QALYs, respectively. In the comprehensive strategy, the average cost was US $\$ 323$ and obtained 18.12 life-years and 17.58 QALYs (Table 2). The immunization strategy cost the least, and the ICER of the comprehensive strategy was US\$35/QALY gained compared to the comparative strategy. The conventional pattern and the treatment strategy were dominated by higher costs but lower benefits compared to the comprehensive strategy (Figure S3, Supplementary file 3).

Cost-Effectiveness Analysis for Various Screening Intensities The costs, QALYs, and ICERs increased with the treatment and comprehensive strategy as the screening intensity increased (Figure 1A, 1B, 1C). However, the cost of the immunization
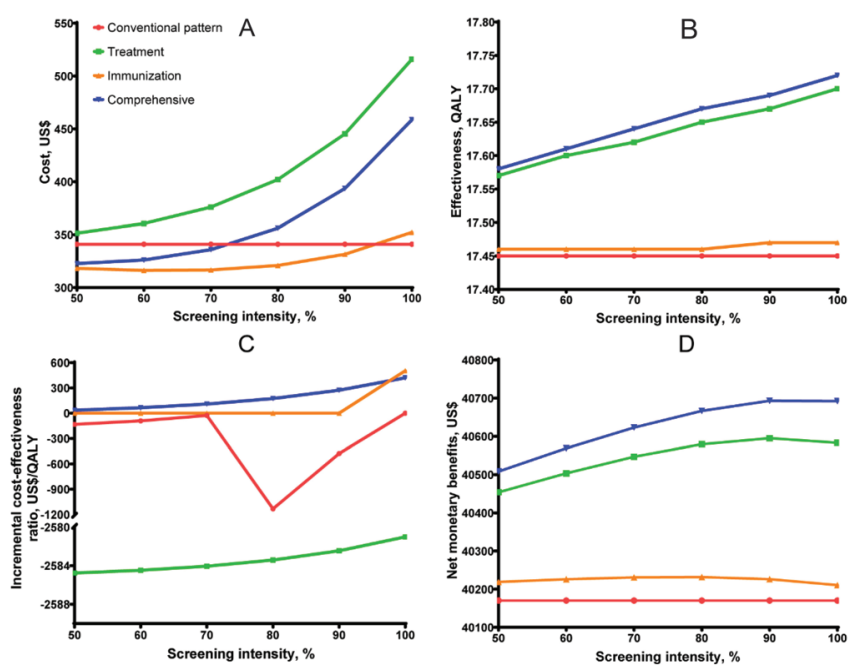

Figure 1. Cost-Effectiveness Analysis Results for Different Screening Intensities. Abbreviation: QALY, quality-adjusted life-year.

strategy tended to decrease first and then increase, but it kept the most cost savings among the four strategies if the intensity was lower than $90 \%$ (Figure 1A). The conventional pattern and treatment strategy were always inferior to the other two strategies as the screening intensity changed (Figure 1C). Except for the conventional pattern, the net monetary benefits of the other three strategies all increased with increasing screening intensity and decreased after 90\% (Figure 1D). The comprehensive strategy gained the most net monetary benefits, with the highest of US\$40693 at the screening intensity of $90 \%$. Compared with the lowest cost strategy, the ICERs of the comprehensive strategy were $65,108,174,272$, and 420 US\$/QALY gained when screening 60\%, 70\%, $80 \%$, $90 \%$, and $100 \%$ of the population, respectively, which were still lower than the WTP (Table S5, Supplementary file 3).

\section{Reduction of Clinical Outcomes}

We established a simulating cohort of $10000 \mathrm{HBV}$ patients and 10000 susceptible individuals to compare the clinical outcomes of the comprehensive intervention and conventional pattern. New infection, compensated cirrhosis, decompensated cirrhosis, HBV-related HCC, liver transplantation, and liver-related mortality would decrease by $50 \%, 26.5 \%, 18.9 \%, 37.6 \%, 31.8 \%$, and $26.2 \%$, respectively, in 2049 if the screening intensity was $50 \%$. The above decreased by $100 \%, 53.2 \%, 39.2 \%, 75.2 \%, 63.6 \%$, and $52.5 \%$, respectively, while the screening intensity reached $100 \%$

Table 2. Summary of Average Outcomes and ICERs at a Screening Intensity of $50 \%$

\begin{tabular}{lcccccc}
\hline \multirow{2}{*}{ Strategy } & \multicolumn{3}{c}{ Average Per Person } & \multicolumn{2}{c}{ Incremental } \\
\cline { 2 - 5 } & Cost, US\$ & QALY & Life-Year & Cost, US\$ & QALY & Life-Year \\
\hline Immunization & 318 & 17.46 & 18.08 & - & - & - \\
Comprehensive & 323 & 17.58 & 18.12 & 4.5 & 0.13 & 0.04 \\
Conventional pattern & 341 & 17.45 & 18.08 & 18.3 & -0.14 & -0.05 \\
Treatment & 351 & 17.57 & 18.12 & 28.7 & -0.01 & 0 \\
\hline
\end{tabular}

Abbreviations: ICER, incremental cost-effectiveness ratio; QALY, quality-adjusted life-year.

The willingness-to-pay threshold is US\$2322 per QALY gained. 
A

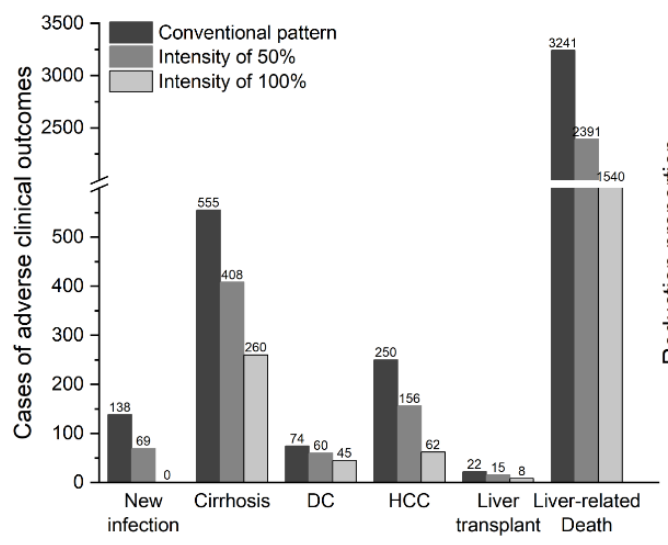

B

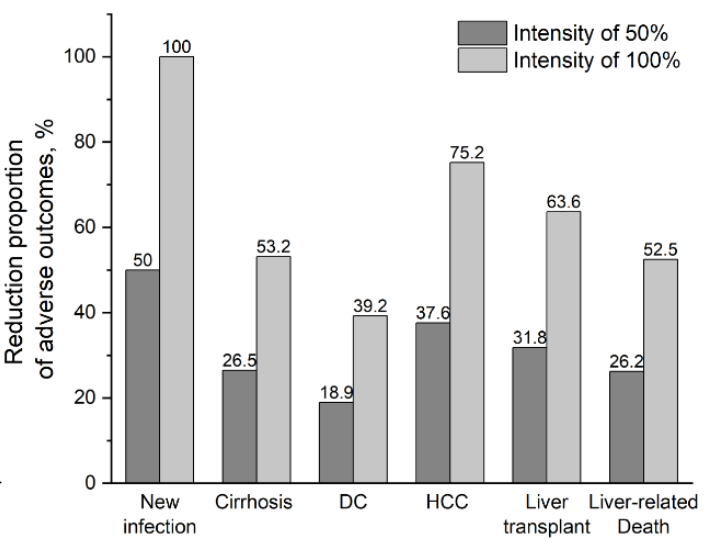

Figure 2. Clinical Outcomes of the Conventional Pattern and Comprehensive Strategy With Different Screening Intensities. (A) Cases of clinical outcomes for the conventional pattern and comprehensive strategy with an intensity of $50 \%$ or $100 \%$; (B) The reduction percentage of clinical outcomes. Abbreviations: DC, decompensated cirrhosis; HCC, hepatocellular carcinoma.

(Figure 2). Fourteen liver transplantations could be avoided for every $10000 \mathrm{HBV}$-infected individuals if population-wide comprehensive intervention was performed.

\section{Sensitivity Analysis}

Figure 1 showed that the comprehensive strategy at a screening intensity of $90 \%$ gained the most net monetary benefit. For this intensity, the ICER was 272US\$/QALY gained for baseline parameters compared to the immunization strategy. Therefore, we displayed the 18 parameters most influential on ICERs at the screening intensity of $90 \%$ in the tornado diagram in Figure 3. The discount rate, screening cost for the comprehensive strategy, natural transition probability from inactive carrier to $\mathrm{CHB}$, and annual $\mathrm{HBs} A g$ clearance rate after antiviral treatment were the four most influential parameters in the model in which the upper limit was more than twice the baseline value. The maximum ICER of the comprehensive strategy was 688US\$/QALY gained if the natural transition probability from inactive carrier to $\mathrm{CHB}$ was $1.6 \%$. Thus, one-way sensitivity analysis showed that the comprehensive strategy was always cost-effective over the wide variety of all parameters.

The results from the two-way sensitivity analysis revealed that the comprehensive strategy always remained costeffective, although the screening costs and intensity varied in the deterministic range (Figure S4, Supplementary file 3).

We then performed 10000 Monte Carlo Simulations and the PSA results were shown in Figure S5 and Figure S6 (see Supplementary file 3 ). The probability that the comprehensive strategy would be cost-effective was $98.4 \%$ at a WTP threshold of 2322US\$/QALY when all parameters were sampled randomly.

\section{Discussion}

Our study suggested that comprehensive intervention, including mass screening, treatment, and immunization,

\begin{tabular}{lccc}
\hline \multirow{2}{*}{ Inputs } & \multirow{2}{*}{ Base case (range) } & \multicolumn{2}{c}{ ICER, US\$/QALY } \\
\cline { 2 - 4 } & Lower limit & Upper lim \\
\hline Discount rate, \% & $3(0-6)$ & 50 & 573 \\
\hline Screening cost for comprehensive, US\$ & $147(73.50-220.50)$ & 38 & 505 \\
\hline Natural probability of IC to CHB, \% & $4.40(1.60-4.70)$ & 249 & 688 \\
\hline Treated probability of IC to HBsAg loss, \% & $4.50(2.00-7.00)$ & 142 & 544 \\
\hline Cost for carrier, US\$ & $75.70(37.80-113.50)$ & 112 & 432 \\
\hline Natural probability of CHB to CC, \% & $3.20(2.40-4.00)$ & 152 & 412 \\
\hline Cost for HCC, US\$ & $2267.20(1133.60-3400.70)$ & 162 & 383 \\
\hline Cost for CC, US\$ & $1252.30(626.20-1878.50)$ & 178 & 366 \\
\hline Natural probability of IC to HBsAg loss, \% & $1.00(0-2.00)$ & 191 & 369 \\
\hline Screening cost for immunization, US\$ & $55(27.50-82.50)$ & 187 & 359 \\
\hline Cost for CHB, US\$ & $205.30(102.60-307.90)$ & 208 & 336 \\
\hline Natural probability of CHB to IC, \% & $5.70(4.58-6.88)$ & 228 & 319 \\
\hline Treated probability of CHB to CC, \% & $1.76(1.33-2.20)$ & 232 & 312 \\
\hline Natural probability of HCC to death, \% & $14.40(11.60-17.20)$ & 239 & 299 \\
\hline Natural probability of DC to death, \% & $26.50(21.40-31.60)$ & 240 & 297 \\
\hline Natural probability of cirrhosis to death, \% & $3.40(2.90-3.90)$ & 251 & 293 \\
\hline Treated probability of CHB to IC, \% & $12.60(11.40-13.80)$ & 255 & 293 \\
\hline Natural probability of CHB to HCC, $\%$ & $0.60(0.53-0.72)$ & 252 & 285 \\
\hline & & & \\
\hline
\end{tabular}

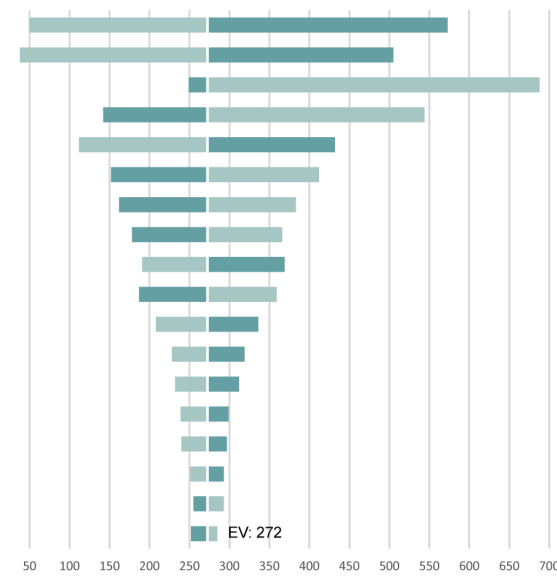

Figure 3. One-Way Sensitivity Analysis of ICERs at a Screening Intensity of $90 \%$ (the Comprehensive Strategy vs. the Immunization Strategy). The willingness-topay threshold is US\$2,322 per QALY gained. The dark green portion of each bar represents the high range of the parameter, and the light green portion of the bar represents the low range of the parameter. When light green is on the left and dark green is on the right, the ICER increases as the parameter value increases. When dark green is on the left, the ICER decreases as the parameter value increases. The white dashed line indicates the ICER for baseline parameters, which is 272 US $\$$ / QALY gained in the figure. Abbreviations: ICER, incremental cost-effectiveness ratios; IC, inactive HBsAg carrier; CHB, chronic hepatitis B; CC, compensated cirrhosis; HCC, hepatocellular carcinoma; DC, decompensated cirrhosis. 
was cost-effective after 30 years of dynamic changes under the current HBV background in rural areas with high prevalence. However, Hutton et al indicated that based on screening and treatment, immunization for close contacts of HBsAg positive patients was more cost-effective than universal immunization (US\$39 903/QALY gained) ${ }^{13}$ Rossi et al reported that the cost of adult immunization was too high and was inferior to the treatment alone strategy. ${ }^{7}$ In regard to the high-risk population, the comprehensive strategy often turned to be cost-effective. For example, Chahal et al found that the screening, treatment, and immunization strategy was cost-effective among six high-risk populations in the United States (US\$3203/QALY gained). ${ }^{12}$ Immunization provided for all HBsAg-negative wives of HBsAg-positive husbands could improve the efficiency of preventing mother-to-child transmission and was cost-effective in China. ${ }^{37}$ Tatar et al compared the cost-effectiveness of universal and targeted hepatitis $C$ virus screening and found that screening and treatment may be cost-effective for people who inject drugs but not for the general population. ${ }^{38}$ However, sensitivity analysis showed that if the treatment cost decreased to $\$ 13200$ per patient and the infection rate increased to $10 \%$, universal screening would be cost-effective. Such differences may be caused by the difference in cost parameters and HBV prevalence in different countries and regions. The centralized drug procedure and use policy in the recent year has caused a sharp reduction in treatment expense for HBV patients, making the comprehensive strategy more affordable and feasible for rural residents. In other words, reducing the medical expense for patients was a stepping stone to eliminating viral hepatitis, which provided a framework for formulating HBV-related health policies in other countries and regions.

Our study set a basis screening intensity of $50 \%$, that is, half of the population was active in participating in the screening. As screening intensity increased, more labor and financial resources were required to convince more people to participate. We quantified this characteristic through exponential screening costs and thereby analyzed the costeffectiveness of various screening intensities, which was not described in other studies. Identifying hidden HBV patients and immunizing susceptible persons are essential methods to inhibit the spread of HBV. Our study indicated that the comprehensive strategy remained cost-effective compared to the other three strategies in high-prevalence and low-income areas. In addition, it had the most net monetary benefit at a screening intensity of $90 \%$ (Figure 1D). Sensitivity analysis revealed that the results remain robust among a wide variety of parameters. Considering that we used the disposable income of rural residents per capita as WTP, our results could be applied in more developed regions.

According to our results, new HBV infections could be eliminated if all susceptible individuals were immunized, but this is impractical. Efforts should be made to achieve immunization coverage over $90 \%$. However, liver-related mortality could only be reduced by $52.5 \%$ with 30 -year normative treatment in 2049. Thus, the efficacy of antiviral drugs must be enhanced to reduce mortality. Recently, scientists were exploring a functional cure, and nucleos $(t)$ ide analogs combined with interferons or sequential therapy were superior to monotherapy in HBsAg loss in a subset of patients. ${ }^{39}$ Besides, other therapeutic strategies, such as the artificial liver support system and liver transplantation, should be employed in severe cases.

We calculated the cost from the perspective of the healthcare payer. Our study carried out an economic evaluation based on the sharp reduction in antiviral drug price and took the screening intensity into account. Different from previous studies, the cost of outpatients was simulated following the treatment and follow-up recommended in the Guideline of Prevention and Treatment for Chronic Hepatitis B in China, therefore reflecting the cost-effectiveness of the comprehensive strategy under standard management regimens. Moreover, we simulated a dynamic transition of HBV in the stable cohort, but population mobility was not considered.

Our study has several limitations. First, we used average costs, utilities, and transition probabilities without considering the effects of age, gender, or comorbidities. Second, the Markov model did not distinguish different HBV genotypes or distinguish $\mathrm{HBV}$ e antigen-positive and HBV e antigennegative CHB. Third, our model ignored consolidation treatment and regular follow-up within one year after the withdrawal of antiviral drugs recommended by the guideline (monthly biochemical routine, HBV serological markers, and HBV DNA quantification in the first three months and once every three months afterward). Finally, we did not consider any adjunctive drugs other than antivirals in the outpatient cost, nor the adverse effects or resistance of antiviral therapy, and the hospitalization cost was obtained from one medical center, but the sensitivity analysis compensated for this to some extent.

Parameters in this model were obtained from published literature and field surveys. The cost-effectiveness results remained robust after we changed the epidemiological parameters and cost parameters, so they can be generalized to other areas of China. Before the intervention strategies were put into practice, we need to consider the affordability for the local government and how to enhance the willingness of residents to participate. Previous practice has shown that combining the HBV screening program with other government policies, such as health examinations, is an approach to increase screening intensity. In addition, with the wide coverage of contracted services by general practitioners in China, we are trying to implement a new HBV management pattern in Zhejiang demonstrated areas, that is, a single-disease contract for HBV to provide specific health management for patients.

\section{Conclusion}

Generally, the strategy of mass HBV screening, immunization for susceptible persons, and treatment for infected patients is of great significance to achieving the goal of eliminating hepatitis B by 2030 and should be put into practice in rural areas with high prevalence. Centralized procurement in China 
has greatly reduced the price of drugs, and this experience could be used in other countries and regions to reduce the disease burden in resource-limited areas and make universal intervention cost-effective.

\section{Ethical issues}

The study has been approved by the Human Research Ethics Committee of the First Affiliated Hospital, College of Medicine, Zhejiang University with informed consent (No. 2017-376).

\section{Competing interests}

Authors declare that they have no competing interests.

\section{Authors' contributions}

Conception and design: BR. Acquisition of data: LJ, CP, and LP. Analysis and interpretation of data: $X X$ and $\mathrm{CW}$. Drafting of the manuscript: XX. Critical revision of the manuscript for important intellectual content: $X Z$ and $B R$. Obtaining funding: BR. Administrative, technical, or material support: WS, LC, and ZL. Supervision: LL, KX, and YD.

\section{Funding}

The work was supported by The National Science and Technology Major Project of China (Grant No. 2017ZX10105001) and National Human Genetic Resources Sharing Service Platform (Grant No. 2005DKA21300).

\section{Authors' affiliations}

'State Key Laboratory for Diagnosis and Treatment of Infectious Diseases, National Clinical Research Center for Infectious Diseases, Collaborative Innovation Center for Diagnosis and Treatment of Infectious Diseases, The First Affiliated Hospital, College of Medicine, Zhejiang University, Hangzhou, China. 2People's Hospital Medical Community of Yuhuan County, Taizhou, China.

\section{Supplementary files}

Supplementary file 1. Markov Model Information.

Supplementary file 2. Epidemiological and Cost Parameters.

Supplementary file 3 contains Table S5 and Figures S3-S6.

\section{References}

1. World Health Organization (WHO). Global Hepatitis Report 2017. Geneva: WHO; 2017.

2. Cui F, Shen L, Li L, et al. Prevention of chronic hepatitis $B$ after 3 decades of escalating vaccination policy, China. Emerg Infect Dis. 2017;23(5):765772. doi:10.3201/eid2305.161477

3. The Polaris Observatory Collaborators. Global prevalence, treatment, and prevention of hepatitis B virus infection in 2016: a modelling study. Lancet Gastroenterol Hepatol. 2018;3(6):383-403. doi:10.1016/s24681253(18)30056-6

4. Nayagam $\mathrm{S}$, Chan $\mathrm{P}$, Zhao $\mathrm{K}$, et al. Investment case for a comprehensive package of interventions against hepatitis B in China: applied modeling to help national strategy planning. Clin Infect Dis. 2021;72(5):743-752. doi:10.1093/cid/ciaa134

5. EASL 2017 Clinical Practice Guidelines on the management of hepatitis B virus infection. J Hepatol. 2017;67(2):370-398. doi:10.1016/j. jhep.2017.03.021

6. Nayagam S, Conteh L, Sicuri E, et al. Cost-effectiveness of communitybased screening and treatment for chronic hepatitis B in The Gambia: an economic modelling analysis. Lancet Glob Health. 2016;4(8):e568-578. doi:10.1016/s2214-109x(16)30101-2

7. Rossi C, Schwartzman K, Oxlade O, Klein MB, Greenaway C. Hepatitis $B$ screening and vaccination strategies for newly arrived adult Canadian immigrants and refugees: a cost-effectiveness analysis. PLOS One. 2013;8(10):e78548. doi:10.1371/journal.pone.0078548

8. Zhang GM, Sun XJ, Wang FZ, Zheng H, Gong XH. Analysis of epidemiological characteristics of hepatitis $B$ among the population of 1859 year old and $\mathrm{HepB}$ immunization strategies. Chin J Vaccines Immun. 2013;19(3):266-270.

9. Pileggi C, Papadopoli R, Bianco A, Pavia M. Hepatitis B vaccine and the need for a booster dose after primary vaccination. Vaccine. 2017;35(46):6302-6307. doi:10.1016/j.vaccine.2017.09.076

10. Hoerger TJ, Schillie S, Wittenborn JS, et al. Cost-effectiveness of hepatitis B vaccination in adults with diagnosed diabetes. Diabetes Care.
2013;36(1):63-69. doi:10.2337/dc12-0759

11. Mangen MJ, Stibbe $\mathrm{H}$, Urbanus A, et al. Targeted outreach hepatitis $B$ vaccination program in high-risk adults: the fundamental challenge of the last mile. Vaccine. 2017;35(24):3215-3221. doi:10.1016/j. vaccine.2017.04.068

12. Chahal HS, Peters MG, Harris AM, McCabe D, Volberding P, Kahn JG. Cost-effectiveness of hepatitis B virus infection screening and treatment or vaccination in 6 high-risk populations in the United States. Open Forum Infect Dis. 2019;6(1):ofy353. doi:10.1093/ofid/ofy353

13. Hutton DW, Tan D, So SK, Brandeau ML. Cost-effectiveness of screening and vaccinating Asian and Pacific Islander adults for hepatitis B. Ann Intern Med. 2007;147(7):460-469. doi:10.7326/0003-4819-147-7200710020-00004

14. Liang $\mathrm{X}, \mathrm{Bi}$ S, Yang $\mathrm{W}$, et al. Epidemiological serosurvey of hepatitis $\mathrm{B}$ in China--declining HBV prevalence due to hepatitis B vaccination. Vaccine. 2009;27(47):6550-6557. doi:10.1016/j.vaccine.2009.08.048

15. Chinese Medical Association. [The guidelines of prevention and treatment for chronic hepatitis B (2019 version)]. Zhonghua Gan Zang Bing Za Zhi. 2019;27(12):938-961. doi:10.3760/cma.j.issn.1007-3418.2019.12.007

16. Fattovich G, Bortolotti F, Donato F. Natural history of chronic hepatitis B: special emphasis on disease progression and prognostic factors. $J$ Hepatol. 2008;48(2):335-352. doi:10.1016/j.jhep.2007.11.011

17. Rendi-Wagner $\mathrm{P}$, Kundi $\mathrm{M}$, Stemberger $\mathrm{H}$, et al. Antibody-response to three recombinant hepatitis $\mathrm{B}$ vaccines: comparative evaluation of multicenter travel-clinic based experience. Vaccine. 2001;19(15-16):2055-2060. doi:10.1016/s0264-410x(00)00410-2

18. Zheng $\mathrm{H}$, Wang $\mathrm{FZ}$, Zhang $\mathrm{GM}$, et al. An economic analysis of adult hepatitis B vaccination in China. Vaccine. 2015;33(48):6831-6839. doi:10.1016/j.vaccine.2015.09.011

19. Hui CK, Leung N, Yuen ST, et al. Natural history and disease progression in Chinese chronic hepatitis B patients in immune-tolerant phase. Hepatology. 2007;46(2):395-401. doi:10.1002/hep.21724

20. Lin X, Robinson NJ, Thursz M, et al. Chronic hepatitis $B$ virus infection in the Asia-Pacific region and Africa: review of disease progression. J Gastroenterol Hepatol. 2005;20(6):833-843. doi:10.1111/j.14401746.2005.03813.x

21. Terrault NA, Bzowej NH, Chang KM, Hwang JP, Jonas MM, Murad MH. AASLD guidelines for treatment of chronic hepatitis B. Hepatology. 2016;63(1):261-283. doi:10.1002/hep.28156

22. Terrault NA, Lok ASF, McMahon BJ, et al. Update on prevention, diagnosis, and treatment of chronic hepatitis B: AASLD 2018 hepatitis B guidance. Hepatology. 2018;67(4):1560-1599. doi:10.1002/hep.29800

23. Tseng TC, Liu CJ, Chen CL, et al. Serum hepatitis B virus-DNA levels correlate with long-term adverse outcomes in spontaneous hepatitis B e antigen seroconverters. J Infect Dis. 2012;205(1):54-63. doi:10.1093/ infdis/jir687

24. Eun JR, Lee HJ, Kim TN, Lee KS. Risk assessment for the development of hepatocellular carcinoma: according to on-treatment viral response during long-term lamivudine therapy in hepatitis $B$ virus-related liver disease. J Hepatol. 2010;53(1):118-125. doi:10.1016/j.jhep.2010.02.026

25. Wong GL, Chan HL, Mak CW, et al. Entecavir treatment reduces hepatic events and deaths in chronic hepatitis B patients with liver cirrhosis. Hepatology. 2013:58(5):1537-1547. doi:10.1002/hep.26301

26. Jang JW, Choi JY, Kim YS, et al. Effects of virologic response to treatment on short- and long-term outcomes of patients with chronic hepatitis B virus infection and decompensated cirrhosis. Clin Gastroenterol Hepatol. 2018;16(12):1954-1963.e1953. doi:10.1016/j.cgh.2018.04.063

27. Kanwal $F$, Hernaez R, Liu $Y$, et al. Factors associated with access to and receipt of liver transplantation in veterans with end-stage liver disease. JAMA Intern Med. 2021;181(7):949-959. doi:10.1001/ jamainternmed.2021.2051

28. Razavi H, Elkhoury AC, Elbasha E, et al. Chronic hepatitis C virus (HCV) disease burden and cost in the United States. Hepatology. 2013; 57(6):2164-2170. doi:10.1002/hep.26218

29. Yang $\mathrm{S}, \mathrm{Yu} \mathrm{C}, \mathrm{Chen} \mathrm{P}$, et al. Protective immune barrier against hepatitis $B$ is needed in individuals born before infant HBV vaccination program in China. Sci Rep. 2015;5:18334. doi:10.1038/srep18334

30. Shepard CW, Simard EP, Finelli L, Fiore AE, Bell BP. Hepatitis B virus infection: epidemiology and vaccination. Epidemiol Rev. 2006;28:112125. doi:10.1093/epirev/mxj009

31. Lok AS, McMahon BJ. Chronic hepatitis B. Hepatology. 2007;45(2):507539. doi:10.1002/hep. 21513 
32. Levy AR, Kowdley KV, lloeje $\mathrm{U}$, et al. The impact of chronic hepatitis $B$ on quality of life: a multinational study of utilities from infected and uninfected persons. Value Health. 2008;11(3):527-538. doi:10.1111/ j.1524-4733.2007.00297.x

33. Chen P, Yu C, Ruan B, et al. Prevalence of hepatitis B in insular regions of southeast China: a community-based study. PLoS One. 2013;8(2):e56444. doi:10.1371/journal.pone.0056444

34. Yu Z, Deng M, Peng C, et al. Asystem dynamics modelling simulation based on a cohort of hepatitis B epidemic research in east China community. Epidemiol Infect. 2019;147:e86. doi:10.1017/s0950268819000220

35. Guilbert JJ. The world health report 2002 - reducing risks, promoting healthy life. Educ Health (Abingdon). 2003;16(2):230. doi:10.1080/1357628031000116808

36. Statistical Communique of the People's Republic of China on the 2019
National Economic and Social Development. National Bureau of Statistics website. http://www.stats.gov.cn/tjsj/zxfb/202002/t20200228_1728913. html. Accessed June 8, 2021. Updated February 28, 2020.

37. Jing W, Liu J, Wu Y, Ma Q, Liu M. Cost-effectiveness of couple-based immunization strategy to prevent mother-to-child transmission of hepatitis $\mathrm{B}$ virus in China: a decision-analytic Markov model. EClinicalMedicine. 2020;19:100264. doi:10.1016/j.eclinm.2020.100264

38. Tatar M, Keeshin SW, Mailliard M, Wilson FA. Cost-effectiveness of universal and targeted hepatitis $C$ virus screening in the United States. JAMA Netw Open. 2020;3(9):e2015756. doi:10.1001/ jamanetworkopen.2020.15756

39. Hou JL, Wei L, Wang GQ, Jia JD, Duan ZP, Zhuang H. [Clinical cure of hepatitis B: consensus and controversy]. Zhonghua Gan Zang Bing Za Zhi. 2020;28(8):636-639. doi:10.3760/cma.j.cn501113-20200722-00409 